\title{
Post-traumatic Growth and Resilience in Adolescents in Puerto Rico Post-hurricane Maria
}

GABRIEL SANTOS MALAVE (D)

CYNTHIA LUO

KANAKO TAKU

ROBERT YANAGISAWA

CRAIG KATZ

*Author affiliations can be found in the back matter of this article

\section{ABSTRACT}

Background/Rationale: Hurricane Maria and the COVID-19 pandemic have greatly impacted the lives of adolescents in Puerto Rico. Understanding how individuals respond to stress can elucidate strategies used to cope and grow from impactful life events (ILE) while minimizing adverse mental health consequences. This expands on work with medical students in Fukushima post-3/11.

Hypothesis or Research Question: Adolescents who utilize more resilience behaviors will experience more post-traumatic growth. Adolescents who demonstrate posttraumatic growth from a previous event will experience less COVID-related stress.

Study Design/Methods: Students were anonymously surveyed and responded to the Davidson-Trauma Scale (DTS), Post-Traumatic Growth Inventory (PTGI), ConnorDavidson Resilience Scale-10 (CDRS-10), Resilient Behavior Scale (RBS), and questions specific to COVID-19 and Hurricane Maria. Data was analyzed by examining correlations between scales and between scales and questions specific to COVID-19 and natural disasters.

Results: Most students indicated the COVID-19 pandemic as their most impactful life event (44\%) (Figure 1). The RBS and CDRS-10 were positively correlated with each other $(r=.679, p=.002)$. The PTGI was positively correlated with the RBS $(r=.878, p$ $<.001$ ) but not the CDRS-10 (Table 1). Students who chose a prior non-COVID-19 ILE experienced less COVID-19 stress $(p=0.047)$ than those who chose COVID-19. Posttraumatic growth (PTG) was greater in those who indicated an ILE affected them and others instead of only them ( $p=0.04$ ) (Table 2). PTG was also positively correlated with finding something positive in Hurricane Maria $(r=.458, p=0.024)$ but not COVID-19. In the free response section, most indicated meeting new people and coming together as positive aspects of Hurricane Maria.
CORRESPONDING AUTHOR:

\section{Gabriel Santos Malave}

Department of Global Health, Icahn School of Medicine at Mount Sinai, US

gabriel.santosmalave@icahn. mssm.edu

KEYWORDS:

COVID-19; Hurricane Maria; natural disasters; global mental health; adolescents; resilience; post-traumatic growth

TO CITE THIS ARTICLE: Santos Malave G, Luo C, Taku K, Yanagisawa R, Katz C. Post-traumatic Growth and Resilience in Adolescents in Puerto Rico Post-hurricane Maria. ISMMS Journal of Science and Medicine. 2021; 1(2): 10, pp. 1-3. DOI: https:// doi.org/10.29024/ijsm.57 
Conclusions/Future Plans: Our results indicate adolescents' resilient behaviors, not resiliency per se, were associated with post-traumatic growth since only RBS was correlated with PTG. Additionally, undergoing prior stressful events appears to mitigate distress from future stressors. Finally, experiencing an ILE alongside others appears to lead to greater PTG suggesting an important role for social connection. The social isolation of COVID-19 may have diffused the potential to find something positive in the event even in those experiencing PTG.

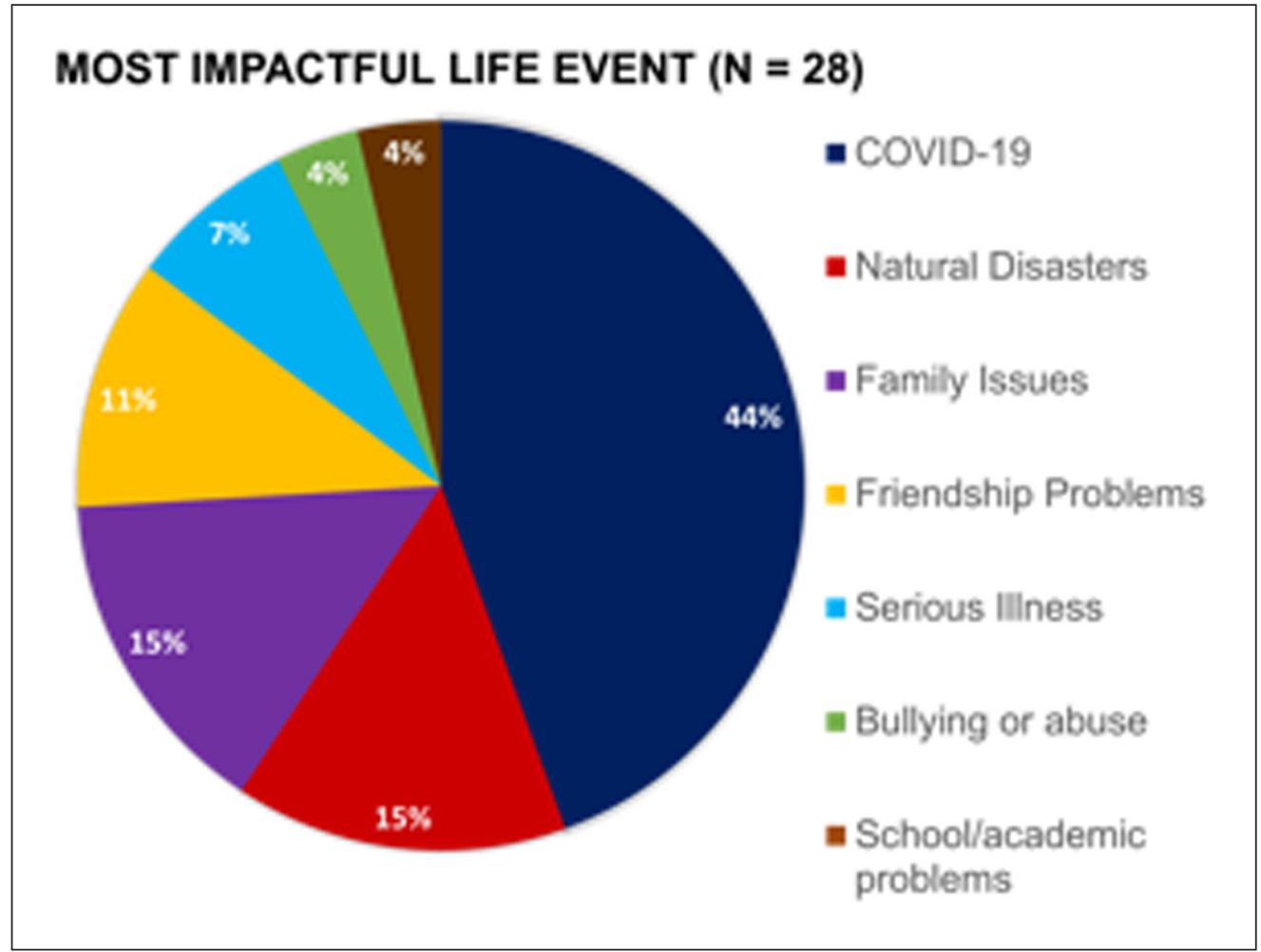

\begin{tabular}{|c|c|c|c|}
\hline \multirow[t]{2}{*}{ A } & & \multicolumn{2}{|l|}{ CDRS-10 } \\
\hline & & PEARSON CORRELATION & $P$ VALUE \\
\hline & PTGI Total & .402 & .052 \\
\hline & RBS & .679 & .002 \\
\hline \multirow{2}{*}{\multicolumn{2}{|c|}{ B }} & \multicolumn{2}{|l|}{ RBS } \\
\hline & & PEARSON CORRELATION & $P$ VALUE \\
\hline & PTGI Total & .878 & $<001$ \\
\hline & PTGI-F1: Relating to Others & .854 & $<001$ \\
\hline & PTGI-F2: New Possibilities & .802 & $<001$ \\
\hline & PTGI-F3: Personal Strength & .706 & 0.001 \\
\hline & PTGI-F4: Spiritual Change & .596 & 0.009 \\
\hline & PTGI-F5: Appreciation of Life & .805 & $<001$ \\
\hline
\end{tabular}

\begin{tabular}{lllll}
\hline & $\begin{array}{l}\text { ILE }=\text { TO YOU } \\
(N=6)\end{array}$ & $\begin{array}{l}\text { ILE }=\text { TO YOU AND } \\
\text { OTHERS }(\mathrm{N}=19)\end{array}$ & TEST & P VALUE \\
\hline PTGI Total & $M=25(16.18)$ & $M=30.83(10.37)$ & -1.111 & 0.046 \\
\hline PTGI-F1: Relating to Others & $M=5.375(4.03)$ & $M=6.7778(2.32)$ & -1.130 & 0.036 \\
\hline PTGI-F2: Nev/Possibilities & $M=5.125(3.94)$ & $M=6.056(2.51)$ & -0.730 & 0.039 \\
\hline
\end{tabular}

Figure $144 \%$ of students indicated COVID-19 as their ILE, 15\% indicated a natural disaster, and $41 \%$ indicated another event.

Table 1 (A) CDRS-10 is positively correlated with RBS but not PTGI; (B) RBS is positively correlated with posttraumatic growth.

Table 2 Students who indicated an ILE that happened to them and others demonstrated significantly greater posttraumatic growth than students who indicated an ILE that only occurred to them. This was driven by PTGI factors 1 and 2 . 
The authors have no competing interests to declare.

\section{AUTHOR AFFILIATIONS}

Gabriel Santos Malave (D) orcid.org/0000-0002-1830-9087

Department of Global Health, Icahn School of Medicine at Mount Sinai, US

\section{Cynthia Luo}

Department of Global Health, Icahn School of Medicine at Mount Sinai, US

\section{Kanako Taku}

Department of Psychology, University of Oakland, US

\section{Robert Yanagisawa}

Department of Global Health, Icahn School of Medicine at Mount Sinai, US

\section{Craig Katz}

Department of Global Health, Icahn School of Medicine at Mount Sinai, US

\section{COPYRIGHT:}

(c) 2021 The Author(s). This is an open-access article distributed under the terms of the Creative Commons Attribution 4.0 International License (CC-BY 4.0), which permits unrestricted use, distribution, and reproduction in any medium, provided the original author and source are credited. See http://creativecommons.org/ licenses/by/4.0/.

ISMMS Journal of Science and Medicine is a peer-reviewed open access journal published by Levy Library Press. 